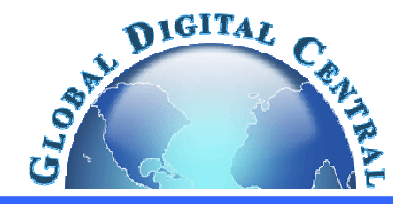

Frontiers in Heat and Mass Transfer

\title{
VISCOUS DISSIPATION EFFECTS ON THE LIMITING VALUE OF NUSSELT NUMBERS FOR A SHEAR DRIVEN FLOW BETWEEN TWO ASYMMETRICALLY HEATED PARALLEL PLATES
}

\author{
Pranab Kumar Mondal, ${ }^{*}$ Sanchayan Mukherjee \\ Department of Mechanical Engineering, Kalyani Government Engineering College, Kalyani - 741235, India
}

\begin{abstract}
The present paper deals with the analytical investigation for the limiting value of Nusselt number, including the effect of viscous dissipation on heat transfer for a laminar shear driven flow between two infinite parallel plates, where the bottom plate is fixed and the top plate is moving in an axial direction at a constant speed. The study concentrates on hydro-dynamically fully developed flow of a Newtonian fluid of constant properties without considering the axial conduction in the fluid. To investigate the effect of viscous dissipation on heat transfer by defining the limiting Nusselt number, plates are kept at constant equal temperatures. Close form expressions for the limiting Nusselt numbers as a function of the Brinkman number and asymmetry parameter are evaluated. Focus is given to the viscous dissipative effect due to the shear produced by the movable top plate over and above the viscous dissipation due to internal fluid friction. The interactive effects of the Brinkman number and the degree of asymmetry on the limiting Nusselt number are investigated analytically. Specific to the cases considered for this study, the appearance of point of singularities due to the variation of Nusselt number with the Brinkman number is observed, and discussion has been made considering the energy balance, and second law analysis of thermodynamics.

Keywords: analytical investigations, heat transfer, shear driven flow, degree of asymmetry, Brinkman number, axial conduction, second law of thermodynamics.
\end{abstract}

\section{INTRODUCTION}

In the recent past, fluid flow in small devices and the corresponding heat transfer has received serious attention in view of the remarkable development in the field of microelectronics and MEMS. The dissimilarity in thermal behavior in small devices is mainly due to the rise in temperature, which is primarily attributable to the viscous dissipation effect. In small devices the effect of viscous dissipation that could play a vital role, results in inefficient heat dissipation leading to local overheating problems. The understanding of the fluid flow phenomena has been critically reviewed (Hak, 1999) through micro scale devices and explored the physics of the plow emphasizing the use of MEMS in different areas for flow control. In the domain of the macroflows, there are so many practical applications where heat transfer normally occurs in the fluid flow system involving moving boundaries. Particularly, in many material processing applications such as extrusion, hot rolling, drawing, and continuous casting, materials continuously move in a channel. In such industrial applications, it is of great importance to encounter the heat transfer from the moving boundary to the surrounding fluid and vice-versa. However, the moving boundary deforms the fluid velocity profile, and shears the fluid layer near the boundary, resulting in local changes in velocity gradient. Thus the viscous dissipation effects may not be neglected in heat transfer analysis associated with moving boundaries. The thermal energy generated due to the viscous dissipation is significant near the wall, which alters the heat transfer rates following the changes in the temperature profile. In order to obtain the actual heat transfer rate in the application of moving boundaries, it is important to take into account the effects of viscous dissipations using accurate velocity distribution. The first theoretical work (Brinkman, 1951) concerning the heat generation due to viscous dissipation has analyzed the effects of viscous heating for the flow of a single phase Newtonian fluid through a circular tube. The temperature distribution in the thermal entrance region has been examined considering the zero temperature of the wall and an insulated wall. The temperatures were found to be the highest, not surprisingly, within a small area near the wall region. The available literature in the area of convective heat transfer has, however, considered the effects of viscous dissipation to be important in two cases: flow of very viscous fluids and flow in capillary tubes. A numerical study (Cheng and $\mathrm{Wu}, 1976$ ) reported the influence of viscous dissipation for the flow of a Newtonian fluid through a parallel plate channel. The numerical analysis explored the onset of the instability for longitudinal vortices in the entrance region of the channel with the thermal boundary condition of an isothermal heated lower plate, and cooled upper-plate. The effects of viscous dissipation on laminar forced convection through a pipe and channel have been studied (Pinho and Oliveira, 2000) for the flow of a Phan- ThienTanner fluid. The study has revealed that the viscous dissipation enhances the fluid elasticity. Performing an analytical study, using a functional analysis method, the effects of viscous dissipation on the heat transfer have been investigated (Lahjomri et al., 2003) for a thermally-developing laminar Hartman flow through a parallel plate channel with the aid of a magnetic field. In a study of thermal development of forced convection in a parallel plate channel filled by porous medium, an investigation of the effects of viscous dissipation has been done (Nield et al., 2003) with the thermal boundary condition of uniform wall temperature including axial conduction effects. 
The analysis of laminar forced convection in a pipe for a Newtonian fluid of constant properties has been performed (Aydin, 2005) by taking the effect of viscous dissipation into account. In Part-1, both hydrodynamically and thermally fully developed convection has been studied, while Part-2 of the study has considered the hydro-dynamically developed but thermally developing case. In both cases, two different types of thermal boundary condition have been considered, namely, constant heat flux (CHF) and constant wall temperature (CWT). The variations of dimensionless radial temperature and Nusselt number have been obtained for different values of Brinkman number under both wall heating and cooling. The influence of viscous dissipation on heat transfer has been found to be strong for higher values of Brinkman number $(\mathrm{Br}>1)$, while the influence has been negligible for lower values of Brinkman number. In the thermally developing case, comparing the temperature distribution with that of the same obtained by neglecting the viscous dissipation, it has been observed that the temperature distribution increases in the axial direction, which is attributable to the effect of viscous dissipations. In their study, an important role of Brinkman number (for the case CWT) and modified Brinkman number (for the case CHF) has also been investigated on the development of the Nusselt number. The analytical work by Aydin and Avci, (Aydin and Avci, 2006) has dealt with the convective heat transfer problem for the plane Poiseuille flow with an emphasis given on the viscous dissipation effect. The energy equation has been solved for thermally developed and developing cases separately with the boundary condition of CWT and CHF, respectively. In both cases, the flow has been considered to be hydro-dynamically developed. It has been found from the study that with the increasing intensity of viscous dissipation (increase in Brinkman number), the heat transfer decreases up to a critical value, and that is attributed to the internal heat generation due to the viscous dissipation effect. In a recent study, (Sheela-Francisca and Tso, 2009) the effects of viscous dissipation on heat transfer between two fixed parallel plates with constant heat flux boundary condition has been reported. The work carried out represents an extended work of Aydin and Avci, (Aydin and Avci, 2006). Various analytical expressions of Nusselt number as a function Brinkman number have been obtained by several researchers as is apparent from the reported investigation. The survey shows the effects of viscous dissipation on laminar heat transfer on a Poiseuille flow in stationary parallel plates for Newtonian as well as non-Newtonian fluids. The steady state laminar heat transfer to a plane Poiseuille-Couette flow of a Newtonian fluid with simultaneous pressure gradient and axial movement of one of the plates has been investigated extensively in reference (Hudson and Bankoff, 1965; Sestak and Rieger, 1969; Bruin, 1972; El-Ariny and Aziz, 1976). The laminar heat transfer in a Couette flow with imposed pressure gradient has been carried out (Lin, 1979) using the power-law model for a non-Newtonian fluid. In all these cases, the energy equation containing the viscous dissipation term has been solved numerically to obtain the effects of viscous dissipation on the heat transfer for the two different thermal boundary conditions of either temperature specified at either the plates, or a specified temperature at the stationary plate with moving plate insulated. Performing a numerical study, the influence of viscous dissipation on fully developed laminar heat transfer has been investigated (Dava et al., 2004) for a non-Newtonian fluid flowing between two parallel plates with the axial movement of one of the plates. However, the study has concentrated on the boundary conditions of constant wall heat flux at one wall with the other insulated, where the focus has been on the viscous dissipation effects.

All the researches mentioned above have dealt with the effect of viscous dissipation on convective heat transfer in a Poiseuille flow for a hydro-dynamically fully developed flow between two parallel plates, considering both the thermally fully developed and developing cases. No work has been reported so far, on the laminar forced convection in the limiting condition, giving the quantitative relation between the different performance index parameters of heat transfer including the viscous dissipation effect for a shear driven flow between two parallel plates kept at constant unequal temperatures.

The objective of the present paper is to analytically probe the combined effects of the Brinkman number and the degree of asymmetry on the temperature profile. To this end, detailed analytical study is carried out to investigate the effect of viscous dissipation on the heat transfer for a shear driven flow for varying degree of asymmetry in the wall heating. Finally, the expressions of the limiting Nusselt numbers are determined from the temperature distribution for the above-mentioned condition.

\section{GOVERNING EQUATIONS AND ANALYSIS OF THE PROBLEM}

The fluid is flowing in the $\mathrm{x}$-direction between two parallel plates where the upper plate is moving with a constant velocity $U_{p}$ whereas the lower plate is fixed. The plates are $2 H$ apart, and the coordinate system is attached with the center line as shown in Fig. 1.

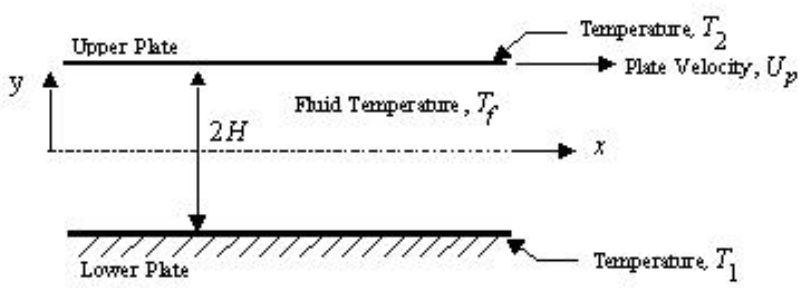

Fig. 1 Schematic Diagram

Following assumptions are made for the analysis:

i) Newtonian fluid;

ii) Incompressible fluid flow;

iii) There is no heat source and thermo-physical properties are constant;

iv) Hydro-dynamically fully developed flow;

v) Axial conduction is neglected in the fluid and through the wall;

vi) Plates are infinitely long in $\mathrm{x}$ and $\mathrm{z}$ directions.

The governing equations are continuity, momentum and energy equations. To get the velocity and temperature distributions between two plates, the governing equations, namely continuity, momentum and energy equations have been derived based on the above-mentioned assumptions.

Continuity Equation

$$
\frac{\partial u}{\partial x}+\frac{\partial v}{\partial y}=0
$$

From the assumptions, there is no velocity in the y-direction, i.e., $v=0$, which gives

$$
\frac{\partial u}{\partial x}=0
$$

Eq. (2) implies that the velocity in the $\mathrm{x}$-direction is a function of $\mathrm{y}$ only.

X-momentum Equation:

$$
\rho\left(u \frac{\partial u}{\partial x}+v \frac{\partial u}{\partial y}+\frac{\partial u}{\partial t}\right)=-\frac{\partial p}{\partial x}+\mu\left(\frac{\partial^{2} u}{\partial x^{2}}+\frac{\partial^{2} u}{\partial y^{2}}\right)
$$

where $p$ is the pressure. Using continuity equation and assumption (iv), one can write the $\mathrm{x}$-momentum equation as follows:

$$
\mu\left(\frac{d^{2} u}{d y^{2}}\right)=\frac{\partial p}{\partial x}+\rho \frac{\partial u}{\partial t}
$$

Now, from y-momentum equation using the above assumptions, it can be shown that 


$$
\frac{\partial p}{\partial y}=0
$$

Energy Equation

$$
\rho C_{p}\left(u \frac{\partial T}{\partial x}+v \frac{\partial T}{\partial y}+\frac{\partial T}{\partial t}\right)=k\left(\frac{\partial^{2} T}{\partial x^{2}}+\frac{\partial^{2} T}{\partial y^{2}}\right)+\mu \phi
$$

where $\phi$ is the viscous dissipation term that contains only $(\partial u / \partial y)^{2}$. Based on the above assumptions, the energy equation reduces to

$$
\rho C_{p}\left(u \frac{\partial T}{\partial x}+\frac{\partial T}{\partial t}\right)=k \frac{\partial^{2} T}{\partial y^{2}}+\mu\left(\frac{\partial u}{\partial y}\right)^{2}
$$

\subsection{STEADY ANALYSIS FOR THE MOVABLE UPPER PLATE WITH A UNIFORM VELOCITY}

The fluid flow is assumed to be due to dragging of the upper plate only. Therefore, Eq. (4) reduces to

$$
\mu\left(\frac{d^{2} u}{d y^{2}}\right)=C
$$

where, $C$ is a constant and this is equal to zero for shear driven flow. No slip condition is assumed at the plates, and thus the boundary conditions are as follows:

at $y=-H, u=0$

and,

$$
\text { at } y=H, u=U_{p}
$$

Solving Eq. (8) with above boundary conditions, the velocity profile is obtained as:

$$
u=\frac{U_{p}}{2}\left(\frac{y}{H}+1\right)
$$

However, defining the non-dimensional quantities $U=\frac{u}{U_{p}}$ and $Y=\frac{y}{H}$, the above velocity profile reduces to,

$$
U=\frac{1}{2}(1+Y)
$$

The energy Eq. (6) is written under steady condition as follows:

$$
\rho C_{p} u \frac{\partial T}{\partial x}=k \frac{\partial^{2} T}{\partial y^{2}}+\mu\left(\frac{\partial u}{\partial y}\right)^{2}
$$

Now to analyze the energy equation in the conduction limit, the following case of unequal constant wall temperatures is considered while neglecting the convection term.

\subsubsection{CONSTANT WALL TEMPERATURE}

For unequal constant wall temperatures, the non-dimensional quantities used as $Y=\frac{y}{H}, U=\frac{u}{U_{p}}$, and defining the dimensionless temperature $\theta=\frac{(T-\bar{T})}{\left(T_{f}-\bar{T}\right)}$, where $T_{f}$ is the initial uniform fluid temperature, the average temperature $\bar{T}=\frac{\left(T_{1}+T_{2}\right)}{2}$, and the asymmetry of wall surface temperature, $\quad \beta=\frac{\left(T_{2}-T_{f}\right)}{\left(T_{1}-T_{f}\right)}$. Again the term $(\partial u / \partial y)^{2}$ is equal to $\left(U_{p} / H\right)^{2}$. Thus the governing equation for constant wall temperatures reduces to,

$$
\frac{d^{2} \theta}{d Y^{2}}+\frac{1}{4} \frac{\mu U_{p}^{2}}{\left(T_{f}-\bar{T}\right) k}=0
$$

Defining the Brinkman number, $B r=\frac{\mu U_{p}^{2}}{k\left(T_{f}-\bar{T}\right)}$, Eq. (14) can be further expressed as

$$
\frac{d^{2} \theta}{d Y^{2}}+\frac{B r}{4}=0
$$

Eq. (15) is subjected to the following boundary conditions:

$$
y=H, \theta=\left(T_{2}-\bar{T}\right) /\left(T_{f}-\bar{T}\right) \text { i.e. at } Y=1, \theta=D
$$

and,

$$
y=-H, \theta=\left(T_{1}-\bar{T}\right) /\left(T_{f}-\bar{T}\right) \text { i.e. at } Y=-1, \theta=-D
$$

Where $D=\frac{(1-\beta)}{(1+\beta)}$

The dimensionless temperature profile is obtained by solving Eq. (15) with the above boundary conditions [Eqs. (16a,b].Therefore, Solving Eq. (15) with the above set of boundary conditions of unequal temperatures, the dimensionless temperature profile in the conduction limit is obtained as:

$$
\theta_{c}=-B r \frac{Y^{2}}{8}+C_{1} Y+C_{2}
$$

The constants $C_{1}$ and $C_{2}$ of Eq. (18), obtained on applying the boundary conditions given in Eq. (16a,b) are as follows:

$$
\left.\begin{array}{l}
C_{1}=D \\
C_{2}=B r / 8
\end{array}\right\}
$$

However, to obtain the expression of Nusselt Number, it is usual to define the mean temperature, $T_{m}$, rather than the centerline temperature in a case of fully developed flow. The mean temperature is given by

$$
T_{m}=\frac{\int_{y=-H}^{H} \rho C_{p} u T_{c} w d y}{\int_{y=-H}^{H} \rho C_{p} u w d y}
$$

where ' $w$ ' is the width of the channel.

The non-dimensional mean temperature is given by

$$
\theta_{m c}=\frac{\left(T_{m c}-\bar{T}\right)}{\left(T_{f}-\bar{T}\right)}=\left(\frac{B r}{12}+\frac{D}{3}\right)
$$

The local heat transfer coefficient in the conduction limit at the lower plate can be evaluated using the equation $q_{1 c}=-\left.k \frac{\partial T}{\partial y}\right|_{y=-H}=h_{1 c}\left(T_{1}-T_{m c}\right)$

Establishing the non-dimensional quantity, Nusselt number in the conduction limit is $N u_{1 c}=\frac{h_{1 c} H}{k}$

However, using Eqs.(19), (22) and (23), the expression for Nusselt number at the lower plate in the conduction limit is given by,

$N u_{1 c}=-\left.\left[1 /\left(\theta_{1}-\theta_{m c}\right)\right]\left(\frac{\partial \theta_{c}}{\partial Y}\right)\right|_{Y=-1}$

Similar to Eqs.(22) to (24), the Nusselt number at the upper plate in the conduction limit can be found to be 


$$
N u_{2 c}=\left.\left[1 /\left(\theta_{2}-\theta_{m c}\right)\right]\left(\frac{\partial \theta_{c}}{\partial Y}\right)\right|_{Y=1}
$$

Where $\theta_{1}, \theta_{2}$ used in Eqs. (24) and (25) are the dimensionless temperatures at the lower plate and the upper plate, respectively. Finally, using Eq. (21) for $\theta_{m}$ and Eq. (18) for the derivative of $\theta_{c}$, the expression of Nusselt numbers on both the plates in the conduction limit are obtained as.

$$
\begin{aligned}
& N u_{1 c}=\frac{3(B r+4 D)}{(16 D+B r)}=\frac{3[B r+4\{(1-\beta) /(1+\beta)\}]}{[16\{(1-\beta) /(1+\beta)\}+B r]} \\
& N u_{2 c}=\frac{3(4 D-B r)}{(8 D-B r)}=\frac{3[4\{(1-\beta) /(1+\beta)\}-B r]}{[8\{(1-\beta) /(1+\beta)\}-B r]}
\end{aligned}
$$

\section{RESULTS AND DISCUSSION}

The Brinkman number is an important parameter governing the heat transfer and fluid flow in a channel between two parallel plates. The effects of viscous dissipation in a fluid flow and heat transfer is manifested by the representation of Brinkman number. Actually, it is a non-dimensional way of representing the effect of viscous dissipation. In this paper, the effect of Brinkman number for a hydro-dynamically fully developed flow has been analyzed. Figures (2) and (3) depict the variation of the dimensionless temperature profile for different Brinkman numbers for two different cases of asymmetric wall heating.

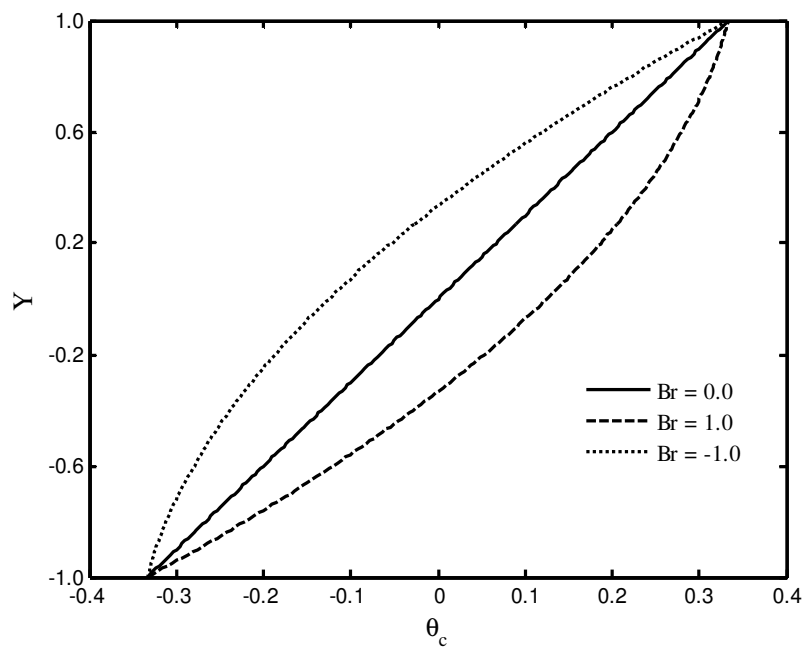

Fig. 2 Dimensionless temperature profile for $\beta=0.5$, for different values of $\mathrm{Br}$

It is observed from Fig. (2) that specific to the case of $\beta=0.5$, the dimensionless temperature $\theta_{c}$ strongly depends on the Brinkman number, $\mathrm{Br}$. Viscous dissipation acts as a source of energy in the flow, and this severely influences the temperature distribution in the flow field as seen from Figs. (2) and (3). In the thermal entrance region, a linear trend of developing dimensionless temperature $\theta_{c}$ is observed for both the case of wall heating for $B r=0$, which is a pure conduction profile. Therefore, in the absence of viscous dissipation, $\theta_{c}$ varies with the specified values of wall surface temperature imposed on the plates. However, viscous dissipation always generates a distribution of heat source stimulating the internal energy in the fluid, and hence the temperature profile gets distorted as it is clear from the Figs. (2) and (3). A close look on the above figures also reveals that the cases with $B r \neq 0$, the profile of the dimensionless temperature gets altered in comparison to that in the case of $B r=0$, though the imposed boundary condition on the plates remain invariant. The reason behind such a behavior of the dimensionless temperature profile obtained at different Brinkman numbers $B r$, is attributable to the effect of viscous dissipation coming into play due to the shear stress within the fluid layer induced by the movement of the upper plate. Positive values of $\mathrm{Br}$ are compatible with the wall heating case, which resembles the situation of heat transfer to the fluid across the wall. Therefore, for the cases with positive values of $\mathrm{Br}$, the fluid temperature increases in comparison to the cases where $B r$ is neglected as evident from Figs. (2) and (3). The reverse holds true for the negative values of $\mathrm{Br}$. Equation (19) predicts the dimensionless temperature distribution in the conduction limit for different values of $\mathrm{Br}$, which is shown in Figs.

(2) and (3). The corresponding Nusselt numbers at both the plates are defined using Eqs. (24) and (25).

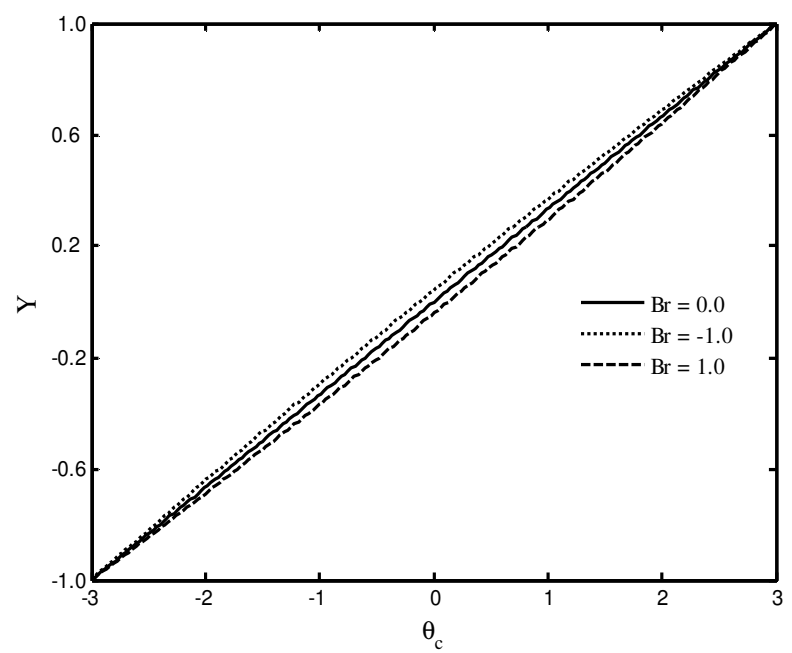

Fig. 3 Dimensionless temperature profile for $\beta=-0.5$, for different values of $\mathrm{Br}$

Figures (4) and (5) are the graphical representation of Nusselt number in the conduction limit on the bottom plate versus Brinkman number $\mathrm{Br}$, specific to the case of asymmetry in the wall heating for $\beta=0.5$ and $\beta=0.0$, respectively. Equations (26) and (27) represent the expression of Nusselt number in the conduction limit on the lower plate and upper plate, respectively. It is observed from Equations. (26) and (27) that both the limiting Nusselt numbers are functions of two independent variables, e.g. the degree of asymmetry in wall heating, $\beta$ and $\mathrm{Br}$. However, both the Nusselt numbers will have parametric variation with $B r$ for $\beta \neq 1$ and with $\beta$ for $B r \neq 0$. As shown, the variation of Nusselt number with $B r$ is not continuous for the case of $\beta=0.5$; rather a singularity is observed at $B r=-5.33$, which is very clear and expected from Eq. (26). At this point, the heat supplied by the wall balances the internal heat generation due to viscous dissipation. However, from this point of singularity with the increasing value of $\mathrm{Br}$ in the positive direction $(B r>0)$, the Nusselt number decreases because of the decrease in the driving potential of the heat transfer, and finally reaches at $N u_{1 c}=3$ asymptotically (when $\mathrm{Br} \rightarrow-\infty$ ). As explained the negative value of $B r$ represents the wall cooling problem and with the increasing value of $\mathrm{Br}$ in the negative direction, Nusselt number decreases and an asymptote appears at $N u_{1 c}=3$ (when $B r \rightarrow-\infty$ ). The result shows that the Nusselt number maintains a constant value as $\mathrm{Br}$ goes to infinity. The expression of Nusselt number in the conduction limit as derived in the study is given in Eq. (26). However, increasing $\mathrm{Br}$ will increase the temperature of the flow field, which, in turn, increases the driving temperature difference of the heat transfer, and hence the Nusselt number might alter. These changes may get reflected on the variation of Nusselt number if the convection term is included in energy equation to obtain the closed-form 
expression for the same. In the limiting condition the effect of increasing $\mathrm{Br}$ is not reflected on the variation of Nusselt number $N u_{1 c}$ for a particular degree of asymmetry. This, however, can also be argued mathematically, from Eq. (26).

Equations (26) and (27) also yield that for any given value of $\beta$, Nusselt number depends on $\mathrm{Br}$ and the limiting values of Nusselt number are not equal (both in magnitude and sign) for given $\beta$. The reason behind such inequality is attributable to the movement of the top plate. The movement of the top plate induces additional shear stress, which enhances viscous heating produced by the internal friction between different fluid layers. However, it is very interesting to notice that when $\mathrm{Br}$ goes to infinity in either direction (i.e. the cold wall and hot wall case), the Nusselt number attains the same asymptotic value, $N u_{1 c}=3$.

Figure (5) also depicts the Nusselt number variation for $\beta=0.0$. The trend observed here can be explained in the similar fashion as in the case with $\beta=0.5$. The only difference noticed for this case is the onset of the point of singularity at $B r=-16$, which can be attributed the effect of the degree in asymmetry in the wall heating. At the point of singularity, the limiting values of Nusselt number approaches a large value for both the cases of asymmetry in wall heating at and, respectively. This is because of the equality in the bulk mean

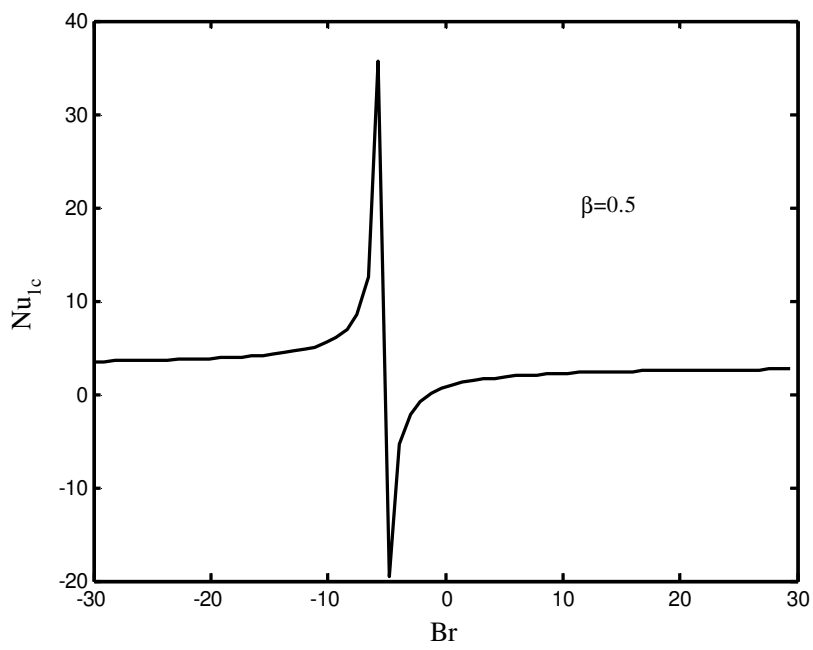

Fig. 4 The influence of $B r$, on the $N u_{1 c}$ for $\beta=0.5$

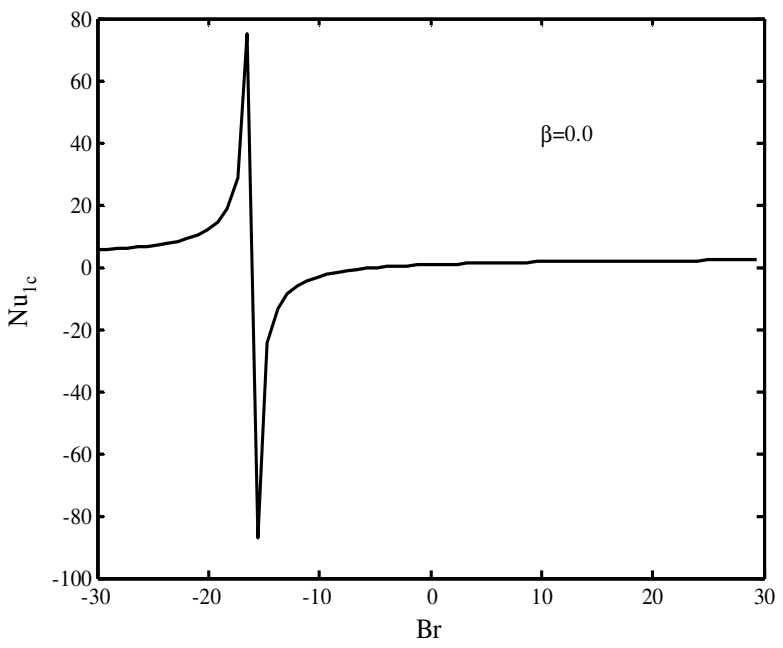

Fig. 5 The influence of $B r$, on the $N u_{1 c}$ for $\beta=0.0$

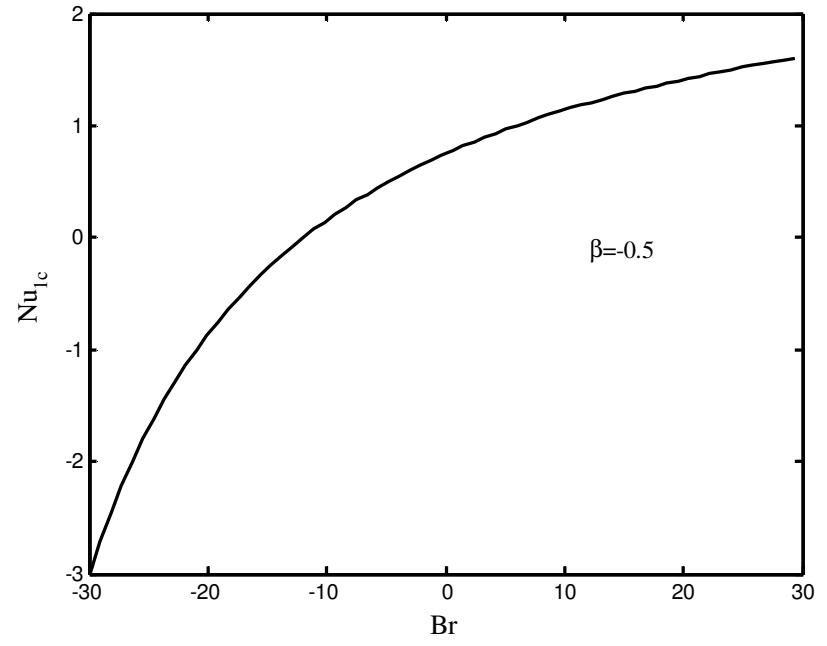

Fig. 6 The influence of $B r$, on the $N u_{1 c}$ for $\beta=0.5$

temperature of the fluid with the average wall surface temperature in the limiting condition. Figure (6) illustrates the effect of $\mathrm{Br}$ on the Nusselt number at the asymmetry in wall heating for $\beta=-0.5$. In contrast to the Figs. (4) and (5), a continuous variation of Nusselt number with $\mathrm{Br}$ is noticed in Fig. (6). This is due to the degree of asymmetry considered in this case. However, the point of singularity is observed to appear at $\mathrm{Br}=-48$, which is an expected result, obtained from Eq. (26) on closely looking into it.

In the present work, heat transfer characteristics in the limiting condition in a viscous dissipative environment are studied thoroughly. The volumetric rate of entropy generation can be expressed as:

$\dot{S}_{g e n}=\frac{k(\nabla T)^{2}}{T^{2}}+\frac{\mu}{T} \phi$

The first term on the right side of the above equation is attributable the irreversibility due to heat transfer and the second term is the entropy generation due to viscous dissipation. Irreversible energy conversion from frictional heating of viscous dissipation into the fluid has an important bearing on the temperature field of the fluid. In the second law analysis, fluid friction irreversibility arises as a result of viscous heating is of essential importance.

Heat transfer dominates for $0 \leq \phi<1$ and fluid friction dominates when $\phi>1$. The contributions of both heat transfer and fluid friction to entropy generation are equal when $\phi=1$.

In the present analysis, three different degrees of asymmetry parameters of wall heating have been considered in investigating the variation of $N u_{1 c}$ as evident from Figs. 4-6. The irreversibility associated with the heat transfer for three different values of asymmetry parameter, $\beta$, however, is related to the irreversibility due to viscous dissipation, and hence different points of onset of singularities are observed for different $\beta$.

\section{CONCLUSIONS}

In the present study, the heat transfer problem for the shear driven laminar flow between two plane parallel plates has been studied. The analysis has been done in the conduction limit, when the plates are kept at unequal constant temperatures. The expression for the Nusselt numbers at both the plates in the conduction limit has been obtained for a hydro-dynamically fully developed flow. After finding the velocity distribution in the flow on solving the momentum equation, it is substituted into the energy equation to obtain the expression of Nusselt numbers. In addition to the effect of viscous dissipation due to the internal fluid friction, an emphasis on viscous dissipation is given to include the effect of shear stress induced by the movement of the top plate. A strong influence of viscous dissipation is observed that is quite significant for analysis of heat transfer in the conduction limit. The 
interactive effects of the Brinkman number and the degree of asymmetry in the wall heating on the limiting values of Nusselt numbers have been investigated in the study. The points of singularities in the variation of Nusselt number versus Brinkman number are also observed. The onset of the points of singularities is seen to be affected by the degree of asymmetry in wall heating, and their sources of appearance have been explained in view of the energy balance, and second law of thermodynamics in the study.

\section{AKNOWLEDGEMENT}

The authors wish to thank Mr. Arijit Dutta, Assistant Professor, Department of Mechanical Engineering of Kalyani Government Engineering College, for his kind help during preparation of the manuscript.

\section{NOMENCLATURE}

\begin{tabular}{|c|c|}
\hline $\begin{array}{l}B r \\
c_{p}\end{array}$ & $\begin{array}{l}\text { Brinkman number } \\
\text { Specific heat at constant pressure }(\mathrm{J} / \mathrm{g} \mathrm{K})\end{array}$ \\
\hline$C_{1}-C_{2}$ & Constants \\
\hline$D$ & Parameter to characterize $(1-\beta) /(1+\beta)$ \\
\hline$h_{1 c}$ & Limiting heat transfer coefficient at lower plate $\left(\mathrm{W} / \mathrm{m}^{2}-\mathrm{K}\right)$ \\
\hline$h_{2 c}$ & Limiting heat transfer coefficient at upper plate $\left(\mathrm{W} / \mathrm{m}^{2}-\mathrm{K}\right)$ \\
\hline$H$ & Half-channel height (m) \\
\hline$k$ & Thermal conductivity $(\mathrm{W} / \mathrm{mK})$ \\
\hline $\mathrm{Nu}$ & Nusselt number \\
\hline$N u_{1 c}$ & Nusselt number in the conduction limit at the lower plate \\
\hline$N u_{2 c}$ & Nusselt number in the conduction limit at the upper plate \\
\hline$t$ & Time (s) \\
\hline$\partial p / \partial x$ & Pressure gradient in the $x$ direction $\left(\mathrm{N} / \mathrm{m}^{3}\right)$ \\
\hline$\partial p / \partial y$ & Pressure gradient in the y direction $\left(\mathrm{N} / \mathrm{m}^{3}\right)$ \\
\hline$q_{1 c}$ & Lower plate heat flux in the conduction $\operatorname{limit}\left(\mathrm{W} / \mathrm{m}^{2}\right)$ \\
\hline$q_{2 c}$ & Upper plate heat flux in the conduction limit $\left(\mathrm{W} / \mathrm{m}^{2}\right)$ \\
\hline$\dot{S}_{g e n}$ & Volumetric rate of entropy generation $\left(\mathrm{W} / \mathrm{m}^{3} \mathrm{~K}\right)$ \\
\hline$T$ & Temperature $(\mathrm{K})$ \\
\hline $\bar{T}$ & Average temperature $(\mathrm{K})$ \\
\hline$T_{1}$ & Upper plate temperature $(\mathrm{K})$ \\
\hline$T_{2}$ & Lower plate temperature $(\mathrm{K})$ \\
\hline$T_{c}$ & Temperature in the conduction limit $(\mathrm{K})$ \\
\hline$u$ & Velocity $(\mathrm{m} / \mathrm{s})$ \\
\hline$U$ & Dimensionless velocity $(\mathrm{m} / \mathrm{s})$ \\
\hline$U_{P}$ & Velocity of the moving plate $(\mathrm{m} / \mathrm{s})$ \\
\hline$x$ & Axial coordinate direction $(\mathrm{m})$ \\
\hline$y$ & Vertical coordinate direction (m) \\
\hline$Y$ & Dimensionless vertical coordinate \\
\hline
\end{tabular}

Greek symbols

$\beta \quad$ Degree of asymmetry

$\theta \quad$ Dimensionless temperature

$\theta_{m} \quad$ Dimensionless bulk mean temperature

$\theta_{m c} \quad$ Dimensionless mean temperature in the conduction limit

$\mu \quad$ Dynamic viscosity $(\mathrm{kg} / \mathrm{m}-\mathrm{s})$

$\rho \quad$ Density $\left(\mathrm{kg} / \mathrm{m}^{3}\right)$

Subscripts

$\begin{array}{ll}c & \text { Conduction limit } \\ f & \text { Initial fluid } \\ m & \text { Mean } \\ m c & \text { Mean in the conduction limit }\end{array}$

\section{REFERENCES}

Aydin, O., 2005, "Effects of Viscous Dissipation on the Heat Transfer in a Forced Pipe. Part 1: Both Hydrodynamically and Thermally Developed Flow," Energy Conversion and Management, 46, 757-69. http://dx.doi.org/10.1016/j.enconman.2004.05.004

Aydin, O., 2005, "Effects of Viscous Dissipation on the Heat Transfer in a Forced Pipe. Part 2. Thermally Developing Flow," Energy Conversion and Management, 46, 3091-3102. http://dx.doi.org/10.1016/j.enconman.2005.03.011

Aydin, O., and Avci, M., 2006, "Viscous-dissipation Effects on the Heat Transfer in a Poiseuille Flow," Applied Energy, 83, 495-512. http://dx.doi.org/10.1016/j.apenergy.2005.03.003

Brinkman, H.C., 1951, "Heat Effects in Capillary Flow I," Appl. Sci. Res, A2, 120-124.

Bruin, S., 1972, "Temperature Distribution in Couette Flow with and without Additional Pressure," Int. J. Heat Mass Transf, 15, 341-349.

Cheng, K.C., and Wu, R-S., 1976, "Viscous Dissipation Effects on Convective Instability and Heat Transfer in Plane Poiseuille Flow Heated from Below," Appl. Sci. Res, 32, 327-46.

Davaa, G., Shigechi, T., and Momoki, S., 2004, "Effects Of Viscous Dissipation On Fully-developed Heat Transfer of Non-Newtonian Fluids in Plane Laminar Poiseuille-Couette Flow," Int. Commun. Heat Mass Transf, 31(5), 663-672.

El-Ariny, A.S., and Aziz., 1976, "Heat Transfer in Plane Couette Flow," Trans. ASME, J. Heat Transfer, 198, 427-431.

Gad-el-Hak, M., 1999, "The Fluid Mechanics of Micro-Devices. The Freeeman Scholar Lecture,” J. Fluids Engg., 121, 5-33. http://dx.doi.org/10.10115/1.2822013

Hudson, J.L., and Bankoff, S.G., 1965, "Heat Transfer to Steady Couette Flow with Pressure Gradient," Journal of Chemical Engineering Science, 20, 415-23.

Lin, S.H., 1979, "Heat Transfer to Plane Non-Newtonian Couette Flow," Int. J. Heat Mass Transf, 22, 1117-1123.

Lahjomri J, Zniber K., and Oubarra, A.A., 2003, "Heat Transfer by Laminar Hartmann's Flow in the Thermal Entrance-region with Uniform Wall Heat-Flux: The Graetz Problem Extended," Energy Conversion and Management, 44, 11-34.

Nield, D.A., Kuznetzov, A.V., and Xiong, M., 2003, "Thermally Developing Forced Convection in a Porous Medium: Parallel-plate Channel with Walls at a Uniform Temperature, with Axial Conduction and Viscous-dissipation Effects," Int. J. Heat Mass Transf, 46, 643-51. http://dx.doi.org/10.1016/S0017-9310(02)00327-7

Pinho, F.T., and Oliveira, P.J., 2000, "Analysis of Forced Convection in Pipes and Channels with the Simplified Phan-thien-tanner Fluid," Int. J. Heat Mass Transfer, 43, 2273-87. http://dx.doi.org/10.1016/S0017-9310(99)00303-8

Sestak, J., and Rieger, F., 1969, "Couette Flow between Parallel Plates," Int. J. Heat Mass Transfer, 12, 71-80.

Sheela-Francisca, J., and Tso, C.P., 2009, "Viscous Dissipation Effects On Parallel Plates with Constant Heat Flux Boundary Condition," Int. Commun. Heat Mass Transf, 36, 249-254.

http://dx.doi.org/10.1016/j.icheatmasstransfer.2008.11.00 